\title{
Hidroxicloroquina no tratamento da Covid-19: uma revisão narrativa
}

\author{
Hydroxychloroquine in the treatment of Covid-19: a narrative review
}

Hidroxicloroquina en el tratamiento de Covid-19: una revisión narrativa

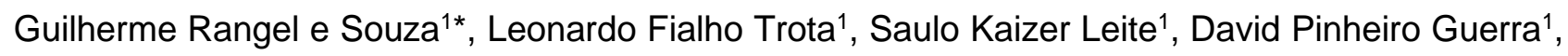
Arthur Pinheiro Santos ${ }^{1}$, Mateus Pimenta Arruda ${ }^{2}$, Lucas do Couto Tonholo ${ }^{3}$, Rosângela Maria de Castro Cunha'.

\section{RESUMO}

Objetivo: Revisar a literatura científica sobre a eficácia e segurança da Hidroxicloroquina (HCQ) no manejo dos pacientes infectados pelo novo coronavírus (SARS-CoV-2), controle da transmissão viral, efeitos adversos e possível uso como profilaxia. Revisão bibliográfica: Observou-se efeito antiviral direto e controle de citocinas em testes in vitro, melhora dos sintomas e diminuição da carga viral em estudos observacionais, nos quadros mais leves da doença. Contudo, divergências no que se refere à segurança do tratamento e a falta de ensaios clínicos randomizados controlados até o momento, não permitem que tal medicamento seja utilizado em larga escala, pois não atendem ao rigor da avaliação da eficácia científica. Considerações finais: São fundamentais ensaios em larga escala de maior impacto científico, a fim de melhor elucidar a eficácia e segurança da HCQ, portanto tem sido recomendada cautela no uso off label desse medicamento para pacientes com COVID-19, a fim de não agravar a condição de saúde.

Palavras-chave: Hidroxicloroquina, Covid-19, Sars-Cov-2, Reações adversas, Tratamento.

\begin{abstract}
Objective: To review the scientific literature on the efficacy and safety of Hydroxychloroquine (HCQ) in the management of patients infected with the new coronavirus (SARS-CoV-2), control of viral transmission, adverses reations and possible use as prophylaxis. Literature review: direct antiviral effect and cytokine control were observed in vitro tests, improvement of symptoms and reduction of viral load in observational studies, in the milder conditions of the disease. However, divergences regarding the safety of treatment and the lack of randomized controlled trials so far, do not allow such a medicine to be used on a large scale, as they do not meet the rigor of the evaluation of scientific effectiveness. Final considerations: Large-scale trials with greater scientific impact are essential in order to better elucidate the efficacy and safety of $\mathrm{HCQ}$, therefore caution has been recommended in the off-label use of this medication for patients with COVID-19, in order not to aggravate the health condition.
\end{abstract}

Keywords: Hydroxychloroquine, Covid-19, Sars-Cov-2, Adverse reactions, Treatment.

\footnotetext{
${ }^{1}$ Faculdade de Ciências Médicas e da Saúde Juiz de Fora (FCMS-JF), Juiz de Fora - MG

*E-mail: guir006@hotmail.com

2 Instituto Penido Burnier, Campinas - SP

${ }^{3}$ Faculdade de Medicina de Barbacena (FUNJOBE), Barbacena - MG
} 


\section{RESUMEN}

Objetivo: Revisar la literatura científica sobre la eficacia y seguridad de la Hidroxicloroquina (HCQ) en el tratamiento de pacientes infectados con el nuevo coronavirus (SARS-CoV-2), control de transmisión viral, su perfil de seguridad y su posible uso como profilaxis. Revisión bibliográfica: se observaron efectos antivirales directos y control de citocinas en pruebas in vitro, mejora de los síntomas y reducción de la carga viral en estudios observacionales, en las condiciones más leves de la enfermedad. Sin embargo, las divergencias con respecto a la seguridad del tratamiento y la falta de ensayos controlados aleatorios hasta el momento, no permita que tal medicamento se use a gran escala, ya que no cumplen con el rigor de la evaluación de la efectividad científica. Consideraciones finales: los ensayos a gran escala con mayor impacto científico son esenciales para dilucidar mejor la eficacia y la seguridad de HCQ, por lo tanto, se recomienda precaución en el uso off-label de este medicamento para pacientes con COVID-19, para no agravar el estado de salud.

Palabras clave: Hidroxicloroquina, Covid-19, Sars-Cov-2, Reacciones adversas, Tratamiento.

\section{INTRODUÇÃO}

Os coronavírus são conhecidos por causarem doenças respiratórias, as quais podem variar de um resfriado a quadros mais graves, incluindo a Síndrome Respiratória do Oriente Médio (MERS) e a Síndrome Respiratória Aguda Grave (SARS). Uma nova cepa foi identificada na cidade de Wuhan, província de Hubei na China em dezembro de 2019, a qual se tornou conhecida por síndrome respiratória aguda grave coronavírus 2 (SARS-CoV-2) causadora da doença de coronavírus 2019 (COVID-19) (ABD EL-AZIZ TM e STOCKAND JD, 2020). O surto inicial na China se espalhou rapidamente por todo o mundo, sendo declarado uma pandemia pela Organização Mundial da Saúde (OMS) em 11 de março de 2020 (AGRAWAL S, et al., 2020).

A SARS-CoV-2 é uma grave ameaça não só à saúde global como também às economias locais, fazendo com que haja uma demanda urgente por medicamentos eficazes, de baixo custo e que possam controlar a pandemia (LIU J, et al. 2020). O contexto atual faz necessário a exigência de terapias que sejam capazes de tratar os pacientes sintomáticos, diminuir a duração do transporte viral e assim evitar a propagação da doença (SUN X, et al., 2020).

Atualmente ainda faltam abordagens eficientes para o controle epidemiológico e para o tratamento do COVID-19, vacinas específicas do genoma de SARS-CoV-2 e anticorpos terapêuticos estão sendo analisados, contudo, essa é uma solução a longo prazo, uma vez que necessitam de testes complexos para validar sua segurança. Dessa forma o redirecionamento para os medicamentos já existentes, ainda que projetados incialmente para outras patologias, passa a ser uma abordagem rápida à pandemia (TU YF, et al.,2020). Essa é uma estratégia plausível, pois já se conhecem os efeitos colaterais, farmacocinética e interações medicamentosas desses agentes (GAUTRET P, et al., 2020).

A inibição da maioria dos coronavírus e inclusive ação antiviral contra a SARS-CoV-1 com o uso de antimaláricos foi relatada anteriormente, assim investigação sobre o efeito da Cloroquina (CQ) / Hidroxicloroquina (HCQ) no SARS-CoV-2 é compreensível (DEVAUX CA, et al., 2020). A HCQ, um derivado da $C Q$, tem mecanismos de ação semelhantes, foi sintetizado pela primeira vez em 1946 demonstrando ser muito menos tóxico que a CQ em estudos nos animais (MCCHESNEY EW, 1983). Além disso, é um medicamento barato e de fácil acesso para o tratamento de doenças autoimunes, portanto é compreensível evocar a ideia da possiblidade de usá-lo no tratamento da COVID-19 (LIU J, et al., 2020).

Antigamente as estratégias terapêuticas para os patógenos visavam principalmente seus genes e proteínas, na maioria das vezes essa abordagem teve êxito, contudo tem muito menos sucesso quando se trata de vírus, pois os genes virais têm como característica sofrer mutações com frequência. Novas medidas para controlar vírus emergentes, incluindo o SARS-CoV-2 devem ser tomadas. SARS-CoV-2 e o SARS-CoV1 usam os receptores da Enzima Conversora de Angiotensina 2 (ACE2) para que assim possam entrar nas 
células, portanto algum tipo de agente que bloqueia essa interação pode ser eficaz no tratamento. Outro fator seria o controle da tempestade de citocinas que ocorre em pacientes com COVID-19 que resultam em pneumonia grave. Dessa maneira direcionar terapêuticas para fatores do hospedeiro pode ser uma abordagem aceitável (LIAO J, et al., 2020).

A CQ e HCQ são medicações primordialmente desenvolvidas para tratamento da malária, porém suas propriedades antivirais e anti-inflamatórias, possibilitaram seu uso de forma diferente do aprovado em bula (off label), especialmente na área da reumatologia. Dessa forma o Food and Drug Administration (FDA) aprovou seus testes contra o COVID-19, uma vez que a HCQ se trata de uma medicação de reposição e baixo custo, sendo uma opção viável para o uso e testes (SAQRANE S e EL MHAMMEDI MA, 2020; ROSA SGV e SANTOS WC, 2020; DONG L, et al., 2020) .

Nos Estados unidos o FDA autorizou o uso de HCQ nos casos de emergência, mas não é uma aprovação desse Agente para tratamento do COVID-19. O que ocorre é um estímulo a elaboração e participação de ensaios clínicos randomizados, para fornecer evidências da eficácia desse medicamento (GANDHI RT, et al., 2020).

Por pressão de parte das de alguns líderes mundiais , mídias sociais e tradicionais , pessoas acabaram induzidas a considerarem a HCQ como cura e até como prevenção da COVID-19, ainda que atualmente não existam ensaios clínicos randomizados e controlados de HCQ no SARS-CoV-1 ou 2, com isso os médicos trabalham em uma situação de escassas evidências (INGRAHAM NE, et al., 2020).

A Organização mundial da saúde (OMS) e o órgão regulador da União europeia (UE) não apoiam a decisão tomada pelas autoridades francesas e americanas de autorizarem o uso da HCQ e CQ. A decisão ignora o procedimento usual de aprovação de medicamentos, que contemplam ensaios clínicos duplo-cegos e controlados por placebo (JAFFE S, 2020).

O Conselho Indiano de Pesquisa Médica (ICMR), recomendou a profilaxia com CQ ou HCQ para profissionais de saúde que tiveram contato com pacientes, ou domiciliares com covid-19 suspeita ou confirmada laboratorialmente. A dose profilática recomendada é de $400 \mathrm{mg}$ duas vezes ao dia no primeiro dia, e $400 \mathrm{mg}$ por semana durante 7 semanas (GUPTA R e MISRA A, 2020).

Isto posto, o objetivo dessa revisão literária é avaliar se a HCQ tem potencial de reduzir o risco de manifestação da doença, curar o paciente infectado, diminuir a disseminação do SARS-CoV-2, investigar os riscos inerentes ao seu uso e a possibilidade de utilizá-lo como profilaxia da COVID-19.

\section{REVISÃO BIBLIOGRÁFICA}

\section{Mecanismos de ação}

A HCQ tem propriedades antivirais de largo espectro, agindo no aumento do pH endossômico, fundamental para a ligação vírus/célula e interferindo na glicosilação dos receptores celulares de SARS-CoV2 (SAQRANE S e EL MHAMMEDI MA , 2020). Além disso, recentemente foi apresentado um novo mecanismo de ação pelo qual a proteína Spike do Sars-CoV-2, na presença de $\mathrm{HCQ} / \mathrm{CQ}$, não consegue se ligar aos gangliosídeos. Pois, a proteína spike viral utiliza não só o receptor da enzima conversora de angiotensina-2 (ACE-2) para se ligar, mas também ácidos siálicos ligados aos gangliosídeos da superfície celular.

Dessa forma sua entrada na célula hospedeira é impedida (FANTINI J, et al., 2020). Exerce efeito antiviral por meio da interferência na glicosilação da ACE2 impedindo a fusão do vírus com a célula hospedeira.

A sinalização dos receptores toll-like (TLR7 e TLR9) e seus ligantes de ácido desoxirribonucleico e ao ácido ribonucleico (RNA/DNA) são suprimidas, da mesma forma a interação entre o DNA citosólico e o monofosfato cíclico de guanosina-adenosina (GMP-AMP) do sensor de ácido nucleico (cGAMP) sintase (cGAS), tal interferência impossibilita a transcrição de genes pró-inflamatórios e com isso haverá menos 
citocinas. A atividade lisossômica em células apresentadoras de antígeno (APCs) é inibida, não ocorrendo o processamento do antígeno e apresentação pelo complexo principal de histocompatibilidade (MHC) classe II para células $T$. Dessa maneira, a ativação de linfócitos $T$ é reduzida e como consequência a diferenciação e expressão de proteínas coestimuladoras fica diminuída (ZHOU D, et al., 2020)

Em investigações anteriores foram detectadas altas concentrações de citocinas séricas nos pacientes em estado crítico, o que sugere uma correlação entre a gravidade da doença e a chamada tempestade de citocinas. A HCQ além do mecanismo antiviral direto, é um agente anti-inflamatório eficaz e seguro com ampla utilização no tratamento de doenças auto-imunes. Logo, pode contribuir para diminuir a resposta inflamatória em pacientes com COVID-19 (LIU J, et al., 2020).

\section{Resultados in vitro}

Nos testes in vitro a CQ e HCQ mostraram ser efetivas contra SARS-CoV-2, com doses clinicamente viáveis, sendo a $\mathrm{HCQ}(\mathrm{EC50}=0,72 \mu \mathrm{M})$ mais potente que a $\mathrm{CQ}(\mathrm{EC50}=5,47 \mu \mathrm{M})$. Baseando-se nos resultados dos modelos PBPK, foi recomendada a dose de $400 \mathrm{mg}$ duas vezes ao dia de sulfato de HCQ a no primeiro dia, seguida de $200 \mathrm{mg}$ duas vezes ao dia, durante 4 dias para infecções pelo COVID-19 (YAO X et al., 2020). Resultados mostram que o HCQ pode inibir eficientemente a infecção por SARS-CoV-2 in vitro. Em combinação com sua função anti-inflamatória acreditamos que o medicamento tenha um bom potencial para combater a doença. Ensaios clínicos vão revelar seu risco-benefício (LIU J, et al., 2020).

\section{Evidências clínicas}

Em um estudo o qual utilizaram CQ em mais de 100 pacientes, obtiveram melhores resultados comparados ao grupo que utilizou uma droga controle, demonstrando eficácia na melhora da imagem pulmonar durante a exacerbação da pneumonia e reduzindo o curso e evolução da doença. (GAO J, et al., 2020).

Gautret $P$, et al. (2020), demonstrou por meio de um estudo observacional, vinte casos tratados por 6 dias com HCQ na dose de $600 \mathrm{mg}$ ao dia, associada a azitromicina tiveram bom resultado na eliminação do vírus comparado ao grupo controle. (70\%) dos pacientes testaram negativo para o Sars-CoV-2 após 6 dias de tratamento, enquanto $(12,5 \%)$ dos pacientes do grupo controle testaram negativo. Os autores indicaram uma significativa eficácia na associação de HCQ e azitromicina comparado ao uso apenas de HCQ, baseado nos testes diários de PCR de swab de nasofaringe, cultura de SARS-CoV-2 e redução precoce da contagiosidade.

Gautret P, et al. (2020), em novo estudo observacional, analisaram 80 pacientes com COVID-19 diagnosticada e que apresentavam em maioria sintomas leves da doença. Foi administrada aos pacientes sem contraindicação, uma combinação de HCQ de $200 \mathrm{mg}$ via oral três vezes ao dia por 10 dias e (500 mg em dia 1, seguida de $250 \mathrm{mg}$ por dia nos próximos quatro dias). Como resultado 78 pacientes apresentaram melhora clínica, além de rápida diminuição da carga viral, (93\%) no dia 8.

Foi demonstrado potencial desse medicamento, tanto para o tratamento dos enfermos com quadros iniciais como para diminuição da disseminação da doença.

Em Marselha França, foram incluídos no estudo retrospectivo 1061 pacientes de faixa etária de 14 a 95 anos, com COVID-19 diagnosticada, os quais receberam HCQ (200 mg três vezes ao dia por dez dias) + AZ (500 mg no dia 1 seguido por $250 \mathrm{mg}$ diariamente nos próximos quatro dias) por pelo menos três dias e acompanhados por 8 dias.

Em sua grande maioria (95\%) apresentavam quadro relativamente leve na admissão e pequena parcela de pacientes com agravamento da doença $(0,9 \%)$. Tal estudo apresentou cura da doença em $(98,7 \%)$ dos pacientes, tendo 8 óbitos de idade média de 79 anos. Foram relatados quadro de hipertensão em 6 desses pacientes, 1 deles apresentava câncer ativo e em 4 foram verificados pneumonia intermediaria a grave, por meio de tomografia computadorizada. Todos esses tiveram avaliações por ECG e nenhum foi confirmada TdP. Os óbitos foram resultantes de insuficiência respiratória. No dia 10 apenas $(4,4 \%)$ persistiram com derramamento viral, fato que pode ter consequências importantes no caráter contagioso da doença, devido ao promissor resultado na depuração viral (MILLION M, et al., 2020). 
Um estudo observacional retrospectivo de Nova York avaliou 1376 pacientes infectados com sarscov2, desses $811(58,9 \%)$ receberam HCQ (600 mg duas vezes no dia 1, depois $400 \mathrm{mg}$ diariamente por uma média de 5 dias) e apresentavam quadro clinico mais grave do que aqueles em que não foi administrada $\mathrm{HCQ}$ $(45,8 \%) .70$ pacientes foram excluídos do estudo por terem necessitado de intubação ou vieram a óbito antes da linha de base do estudo. Como resultado $(25,1 \%)$ dos pacientes desenvolveram insuficiência respiratória, 180 necessitaram de intubação e 166 morreram sem intubação. Não foi revelado maior ou menor risco de intubação, ou morte nos pacientes que receberam HCQ e devido ao desenho observacional e o intervalo de confiança relativamente amplo, o presente estudo não deve ser usado para desconsiderar os benefícios da HCQ nem os riscos de seu uso. Portanto, são imprescindíveis ensaios clínicos randomizados (GELERIS J, et al., 2020).

\section{Avaliação dos efeitos adversos}

No ponto de vista dos reumatologistas a dosagem segura de sulfato de HCQ é de 6-6,5 $\mathrm{mg} / \mathrm{kg}$ por dia, o que poderia gerar níveis séricos de 1,4-1,5 $\mu \mathrm{M}$ em humanos. Por ser uma medicação com baixo nível de acúmulo no tecido, a dose máxima tolerável para HCQ a é de $1200 \mathrm{mg}$, logo, $400 \mathrm{mg}$ diários estão em uma faixa de dosagem segura. Pacientes submetidos a usos prolongados da medicação nessa dose segura, apresentariam poucos efeitos colaterais (SUN X, et al., 2020).

A CQ e a HCQ são medicações toxicas em altas doses, levando ao rápido início de toxicidade do sistema nervoso central (convulsões e coma) além de insuficiência cardiovascular, e retinopatias (KUMAR GV, et al.,2020).

A presença da molécula hidroxila faz com que a $\mathrm{HCQ}$ seja menos permeável à barreira sanguínea da retina com menor risco de toxicidade em comparação com a CQ. Além disso, foi constatado margem estreita entre dosagem terapêutica e toxica da CQ, fazendo com que a HCQ seja uma droga mais segura (SINGH AK, et al., 2020). Há décadas já se tem conhecimento do risco de retinopatia associada ao uso de HCQ, em doses terapêuticas.

Entretanto, esse risco é maior em pacientes tratados por períodos prolongados. Não há necessidade de uso por longos períodos como medicamento pós-exposição ao covid-19 (PICOT S, et al., 2020). Baseado nesses ensaios clínicos pequenos e não controlados, a combinação de HCQ e azitromicina se tornou a primeira opção no tratamento do COVID-19, entretanto os riscos como prolongamento do segmento QT, Torsades de Pointes (TdP) e morte cardíaca súbita não foram avaliados (SARAYANI A, et al., 2020).

É necessário considerar o potencial risco de prolongamento do segmento QT pelo uso de HCQ ou pela combinação HCQ e Azitromicina, no entanto, o esse efeito colateral é raro e alterações cardíacas como a miocardite podem ser ocasionadas pelo próprio SARS-CoV-2. Portanto, nos pacientes portadores de doenças cardiovasculares subjacentes o HCQ deve ser evitado e o exame eletrocardiograma (ECG) deve ser realizado anteriormente ao início do tratamento, assim como a monitorização pelo ECG pode ser utilizada, caso seja necessário (SUN X, et al., 2020).

O prolongamento do intervalo QT induzido por drogas há muito tempo serve como um indicador do aumento do risco de $\mathrm{TdP}$, uma taquicardia ventricular polimórfica potencialmente letal. $\mathrm{O}$ alto risco de morte súbita e arritmia maligna tem o prolongamento corrigido do intervalo QT (QTc) absoluto> 500ms como um dos marcadores importantes (CHORIN E, et al., 2020).

Pacientes com covid-19 ao receberem tratamento com HCQ frequentemente apresentaram prolongamento de QTc, principalmente aqueles nos quais foi feita a combinação HCQ e azitromicina. Foi relatado 1 caso de TdP em paciente que recebeu essa combinação.

Contudo, pela ausência de um Braço controle, não foi possível concluir de fato se a HCQ e Azitromicina tem maior risco cardiotóxico, embora tenham contribuído para os efeitos adversos observados. Também não se pode excluir pacientes com comorbidades cardíacas subjacentes são desproporcionalmente afetados pelo COVID-19 e o próprio vírus tem capacidade de provocar lesões ao miocárdio (MERCURO NJ, et al., 2020). 
Ao avaliar 251 pacientes com COVID-19 tratados com a combinação de HCQ e azitromicina, foi encontrado uma quantidade significativa de prolongamento de QTc (23\%) e um caso suspeito de $\operatorname{TdP}(0,4 \%)$, o qual necessitou de cardioversão. 7 pacientes tiveram termino prematuro da terapia, pois apresentaram extremo prolongamento da QTC.

Esse resultado pode ser explicado pelas características específicas da população contemplada pelo estudo, a qual possui outros fatores de risco para TdP, incluindo fatores genéticos, presença de cardiopatia subjacente, insuficiência renal aguda e coadministração de medicamentos que prolongam a QT, em especial a Amiodarona (CHORIN E, et al., 2020)

Até o presente momento do artigo, não há evidências suficientes que reiteram doses seguras de HCQ e $\mathrm{CQ}$ em monoterapia para pacientes no combate ao Sars-CoV-2. Os estudos que fundamentaram os intervalos de segurança em pacientes, usaram a medicação em condições recorrentes, como lúpus e sinusite. Sendo assim tem-se uma lacuna para que futuras pesquisas e ensaios clínicos tragam dados necessários para se entender a eficácia da medicação, tanto no tratamento quanto na prevenção do COVID-19 (SARAYANI A, et al., 2020).

É imprescindível que as análises de segurança estejam atentas nas alterações dos pacientes, principiantes quanto ao prolongamento do seguimento QT, o qual pode estar mais presente em pacientes críticos com insuficiência hepática ou renal, distúrbios eletrolíticos e outras condições que favorecem o óbito (SARAYANI A, et al., 2020).

Os primeiros agentes terapêuticos reaproveitados com resultados positivos em ensaios clínicos contra SARS-CoV-2 foram a HCQ combinada com azitromicina. Porém, há necessidade de estudos mais amplos, contemplando maior número de pacientes, para validar esses resultados positivos (ROSA SGV e SANTOS WC, 2020).

Recentemente resultados positivos referente a utilização de $\mathrm{HCQ}$, apesar de valiosos, não oferecem evidências satisfatórias para definir a atividade farmacológica ou biológica desse medicamento com probabilidade de ser terapeuticamente útil, isso se deve ao fato da maioria desses estudos serem observacionais e não controlados (PICOT S, et al., 2020).

Em locais sobrecarregados pela doença e com recursos limitados o uso de HCQ pode ser uma estratégia eficaz, pelo fato da escassez de outras opções válidas e o baixo custo dessa medicação. Embora as evidências da efetividade da HCQ sejam limitadas (SINGH AK, et al., 2020).

A CQ e a HCQ não são usadas como profilaxia do COVID-19, isso porque há efeitos colaterais frequentes associados ao seu uso, como piora da visão, náusea, distúrbios digestivos e em casos mais graves podem gerar insuficiência cardíaca (ABD EL-AZIZ TM e STOCKAND JD, 2020).

Não existem evidências que corroborem para uso profilático de HCQ, desse modo não devem ser usados para esse fim, caso contrário podem ocasionar privação desse medicamento para o tratamento de outras patogenias como as doenças reumáticas.

E um alerta deve ser feito quanto aos comentários de líderes mundiais, os quais muitas vezes são prematuros quanto a verdadeira eficácia do tratamento (INGRAHAM NE, et al., 2020). Atualmente há vários estudos randomizados em andamento para avaliar o uso profilático da $\mathrm{HCQ}$, no entanto, pela falta até então de resultados quanto a essa finalidade em especial, não há recomendação para o uso de HCQ nesse sentido (AGRAWAL S, et al., 2020).

\section{CONSIDERAÇÕES FINAIS}

A HCQ possui grande potencial no tratamento da COVID-19, apresentou resultados positivos em estudos in vitro e observacionais, ao que parece tem maior eficácia em quadros mais leves da doença, nos quais apresentou desfechos satisfatórios, enquanto nos pacientes graves não foi possível estabelecer efeito 
benéfico de seu uso. A respeito do uso profilático da $\mathrm{HCQ}$, não foi encontrado em nosso estudo nenhuma evidência que justifique seu uso. Os efeitos colaterais da medicação especificamente em pacientes com COVID-19 ainda são incertos. A principal crítica feita aos estudos observacionais se refere à falta de ensaios randomizados não controlados. Dessa forma, pesquisas de maior impacto científico se fazem necessárias, antes que se possa utilizar esse agente terapêutico em larga escala e com segurança.

\section{REFERÊNCIAS}

1. ABD EL-AZIZ TM, STOCKAND JD. Recent progress and challenges in drug development against COVID-19 coronavirus (SARS-CoV-2) - an update on the status. Infection, Genetics and Evolution, 2020; 83: 1043272.

2. AGRAWAL S, et al. Emerging prophylaxis strategies against COVID-19. Monaldi Archives for Chest Disease, 2020; 90(1): 169-172.

3. CHORIN E, et al. QT Interval Prolongation and Torsade De Pointes in Patients With COVID-19 Treated with Hydroxychloroquine/Azithromycin. Heart Rhythm,2020; S1547-5271(20)30435-5.

4. DEVAUX CA, et al. New insights on the antiviral effects of chloroquine against coronavirus: what to expect for COVID19? International Journal of Antimicrobial Agents,2020;55(5):105938

5. DONG L et al. Discovering Drugs to Treat Coronavirus Disease 2019. Drug discoveries \& therapeutics, 2020; 14(1):5860.

6. FANTINI J, et al. Structural and molecular modelling studies reveal a new mechanism of action of chloroquine and hydroxychloroquine against SARS-CoV-2 infection. International Journal of Antimicrobial Agents, 2020;55(5):105960.

7. GANDHI RT, et al. Mild or Moderate Covid-19. The New England Journal of Medicine, 2020;32329974.

8. GAO J et al. Breakthrough: chloroquine phosphate has shown apparent efficacy in treatment of COVID-19 associated pneumonia in clinical studies. Bioscience trends, 2020;14(1):72-73.

9. GAUTRET $P$, et al. Clinical and microbiological effect of a combination of hydroxychloroquine and azithromycin in 80 COVID-19 patients with at least a six-day follow up: A pilot observational study. Travel Medicine and Infectious Disease, 2020; 34: 101663.

10. GAUTRET P, et al. Hydroxychloroquine and azithromycin as a treatment of COVID-19: results of an open-label nonrandomized clinical trial. International Journal of Antimicrobial Agents, 2020;105949.

11. GELERIS J, et al. Observational study of hydroxychloroquine in hospitalized patients with Covid-19. New England Journal of Medicine, 2020; NEJMoa2012410.

12. GUPTA R, MISRA A. Contentious issues and evolving concepts in the clinical presentation and management of patients with COVID-19 infection with reference to use of therapeutic and other drugs used in Co-morbid diseases (Hypertension, diabetes etc). Diabetes \& Metabolic Syndrome: Clinical Research \& Reviews, 2020; 14(3): 251-254.

13. INGRAHAM NE et al. Shining a light on the evidence for hydroxychloroquine in SARS-CoV-2. Critical Care, 2020; 24: 182.

14. JAFFE S. Regulators split on antimalarials for COVID-19. The Lancet,2020; 395: 1179.

15. KUMAR GV, et al. A short review on antibody therapy for COVID-19. New Microbes and New Infections, 2020; 35: 100682.

16. LIAO J et al. Target Virus or Target Ourselves for COVID-19 Drugs Discovery? —Lessons learned from anti-influenza virus therapies. Medicine in Drug Discovery, 2020; 5: 100037.

17. LIU J et al. Hydroxychloroquine, a less toxic derivative of chloroquine, is effective in inhibiting SARS-CoV-2 infection in vitro. Cell Discovery, 2020; 6:16.

18. MCCHESNEY EW. Animal Toxicity and Pharmacokinetics of Hydroxychloroquine Sulfate. The American journal of medicine, 1983; 75: 11-18.

19. MERCURO NJ et al. Risk of QT Interval Prolongation Associated with Use of Hydroxychloroquine with or Without Concomitant Azithromycin Among Hospitalized Patients Testing Positive for Coronavirus Disease 2019 (COVID-19). JAMA Cardiology, 2020; e201834.

20. MILLION M, et al. Early Treatment of COVID-19 Patients with Hydroxychloroquine and Azithromycin: A Retrospective Analysis of 1061 Cases in Marseille, France. Travel Medicine and Infectious Disease, 2020; 101738.

21. PICOT S, et al. Coalition: Advocacy for prospective clinical trials to test the post-exposure potential of hydroxychloroquine against COVID-19. One Health, 2020; 9: 100131.

22. ROSA SGV, SANTOS WC. Clinical trials on drug repositioning for COVID-19 treatment. Revista panamericana de salud publica, 2020; 44: e40. 
23. SAQRANE S, EL MHAMMEDI MA. Review on the global epidemiological situation and the efficacy of chloroquine and hydroxychloroquine for the treatment of COVID-19. New Microbes and New Infections, 2020; 35: 100680.

24. SARAYANI A et al. Safety signals for QT prolongation or Torsades de Pointes associated with azithromycin with or without chloroquine or hydroxychloroquine. Research in Social and Administrative Pharmacy, 2020;32327397.

25. SINGH AK, et al. Chloroquine and hydroxychloroquine in the treatment of COVID-19 with or without diabetes: $A$ systematic search and a narrative review with a special reference to India and other developing countries. Diabetes \& metabolic syndrome, 2020; 14(3): 241-246.

26. SUN X, et al. Rheumotologitsts' view on the use of hydroxychloroquine to treat COVID-19. Emerging Microbes \& Infections, 2020; 9(1): 830-832.

27. TU YF, et al. A Review of SARS-CoV-2 and the Ongoing Clinical Trials. International journal of molecular sciences, 2020; 21 (7): 2657.

28. YAO X, et al. In Vitro Antiviral Activity and Projection of Optimized Dosing Design of Hydroxychloroquine for the Treatment of Severe Acute Respiratory Syndrome Coronavirus 2 (SARS-CoV-2) Clinical Infectious Diseases, 2020; ciaa237.

29. ZHOU D, et al. COVID-19: a recommendation to examine the effect of hydroxychloroquine in preventing infection and progression. The Journal of antimicrobial chemotherapy, 2020; dkaa169. 